\title{
Einige neue Abkömmlinge des Dioxindols
}

von

\section{Moritz Kohn und Alfons Ostersetzer.}

Aus dem II. chemischen Universitätslaboratorium in Wien und aus dem Laboratorium der Wiener Handelsakademie.

(Vorgelegt in der Sitzung am 6. Juli 1911.)

Durch Einwirkung magnesiumorganischer Verbindungen auf das Isatin sind von dem einen von uns ${ }^{1}$ substituierte Dioxindole mit tertiärer Hydroxylgruppe erhalten worden. Es ist seinerzeit das Phenylderivat, das $\alpha$-Naphthylderivat, das Benzylderivat und das $p$-Bromphenylderivat beschrieben worden. Es ist nunmehr auch gelungen, das 3-Methylderivat des Dioxindols (I) durch Behandlung des Isatins mit Magnesiummethyljodid zu gewinnen.

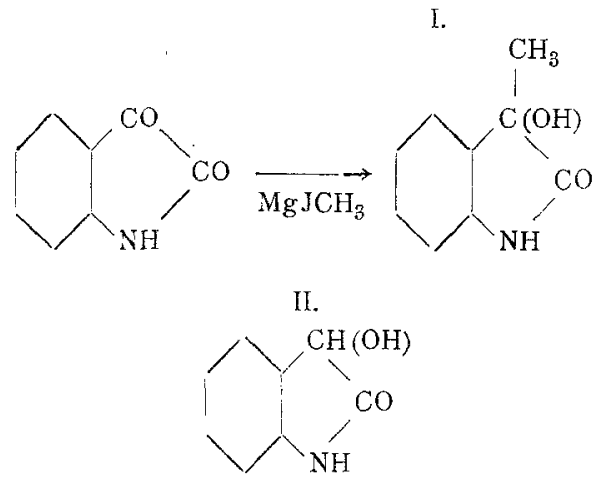

Wie a priori zu erwarten stand, weist dieses 3-Methyldioxindol mit dem Dioxindol (II) eine weitgehende Ähnlichkeit

1 M. Kohn, Monatshefte für Chemie, 1910, 747 u. f. 
auf. Es liefert ebenso wie das Dioxindol schon mit Bromwasser in der Kälte ein Monobromprodukt. Die Grignard'sche Reaktion läßt sich auch auf das 5-Bromisatin (III) übertragen.

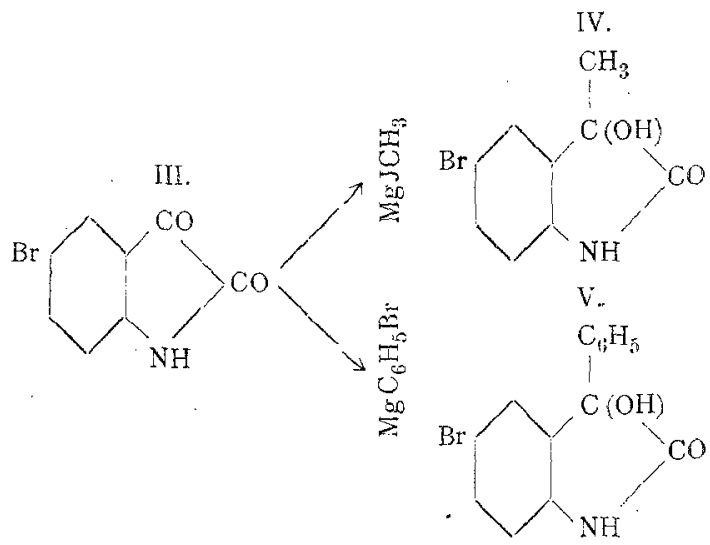

Dasselbe gibt bei der Einwirkung von Magnesiummethyijodid das 3-Methyl-5-bromdioxindol (IV), bei der Einwirkung von Magnesiumphenylbromid das 3-Phenyl-5-Bromdioxindol(V).

Durch Methylierung mittels Dimethylsulfat und Kali lassen sich die substituierten Dioxindole mit tertiärem Hydroxyl, wie durch eine Reihe von Analysen erwiesen wurde, in Dimethylderivate überführen. Diese Methylierungsprodukte enthalten das eine Methyl gebunden an, Sauerstoff, da sie in Zeisel's Apparat glatt ein Methoxyl abspalten. Das zweite bei der Methylierung eingetretene Methyl muß demgemäß am Stickstoff haften:

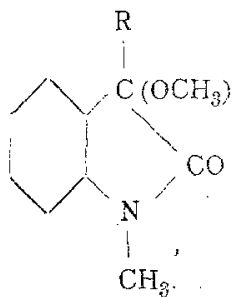

Eine quantitative Bestimmung des Methyls am Stickstoff nach Herzig-Meyer hat sich als nicht ausführbar erwiesen. 
Daß Dimethylsulfat bei Gegenwart von Alkali auch alkoholisches Hydroxyl veräthern kann, erscheint uns besonders bemerkenswert.

Die Struktur des bereits erwähnten Bromproduktes des Methyldioxindols konnte dadurch festgestellt werden, daß es sich als identisch erwies mit dem Brommethyldioxindol (IV), welches durch Behandlung des Bromisatins mit Magnesiummethyljodid entsteht. Die auf diesen beiden Wegen erhaltenen Brommethyldioxindole zeigten, wie dem experimentellen Teile des näheren $z u$ entnehmen ist, den gleichen Schmelzpunkt. Auch der Mischschmelzpunkt beider Substanzen zeigte keine Depression. Zur weiteren Identifizierung wurden die beiden Brommethyldioxindole verschiedener Provenienz mit Kali und Dimethylsulfat methyliert. Die so gewonnenen Methylierungsprodukte besaßen genau den gleichen Schmelzpunkt, ihr Gemenge schmolz ohne eine Spur einer Depression. Daraus geht also hervor, daß bei der direkten Bromierung des Methyldioxindols das Brom in die Parastellung zur Imidogruppe tritt:

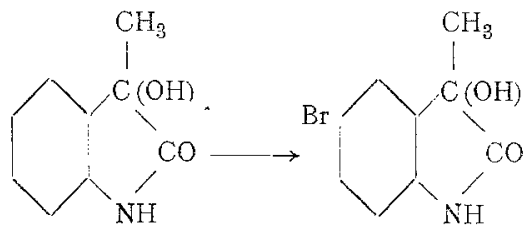

Diese Beobachtung entspricht völlig dem Verhalten des Isatins, welches bei der Bromierung in wässeriger Suspension bekanntlich das 5 -Bromisatin (III) liefert.

Aus unseren Ergebnissen kann man mit großer Wahrscheinlichkeit folgern, daß auch die Bromierung des Dioxindols sich in gleicher Weise abspielt. Man müßte demnach in dem von Baeyer und Knop ${ }^{1}$ entdeckten Bromdioxindol, welches bei der Einwirkung von Bromwasser auf wässerige Dioxindollösung entsteht, das Brom desgleichen in Parastellung zur Imidogruppe annehmen: 


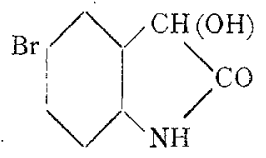

Das Phenyldioxindol läßt . sich durch Acetylierung mit Essigsäureanhydrid in ein Monoacetylprodukt überführen. Wir glauben demselben die Struktur eines N-Acetylproduktes zuschreiben zu müssen:

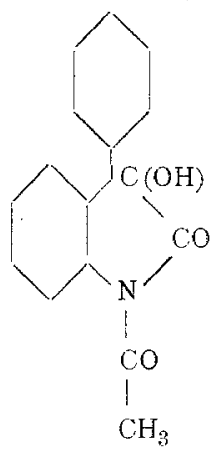

Beim Methyldioxindol hat die Acetylierung, wiewọl sie unter genau den gleichen Bedingungen vorgenommen wurde, wie beim Phenylkörper zum Diacetat geführt.

Das tertiäre Hydroxyl könnte hiernach durch den daran gebundenen Phenylrest für die Acetylierung als sterisch behindert erscheinen. Übrigens hat Suida ${ }^{1}$ aus dem Dioxindol durch Acetylierung vor einer Reihe von Jahren desgleichen ein Monoacetylprodukt (N-Acetyldioxindol) erhalten, während bei der Benzoylierung des Dioxindols Heller ${ }^{2}$ je nach den Versuchsbedingungen ein Mono-, bezichungsweise Dibenzoylderivat erhalten konnte.

Bei der Darstellung der Dioxindole hat es sich als zweckmäßig erwiesen, das seinerzeit angegebene Verfahren ${ }^{3}$ etwas zu modifizieren. Das Isatin wurde nicht wie früher in pulverigem, trockenem Zustand in die Grignardlösung eingetragen, sondern

1 Berl. Ber., 12, 1326.

2 Berl. Ber., 37, 938 u. f.

3 M. Kohn, Monatshefte für Chemie, 1910, 749. 
zuerst mit Äther angerührt und in Form dieser ätherischen Suspension in die Ätherlösung der betreffenden magnesiumorganischen Verbindung einlaufen gelassen. Man vermeidet auf diese Weise, daß größere Mengen Isatins sich der Umsetzung entziehen und das Rohprodukt verunreinigen. Es wurde ferner das Rohprodukt stets zuerst mit einer zur Lösung unzureichenden Menge stark verdünnter Kalilauge bei Wasserbadtemperatur kurze Zeit digeriert. Hierbei gehen hauptsächlich unverändertes Isatin und färbende Verunreinigungen in Lösung, während die Hauptmenge des Dioxindols zurückbleibt. Dasselbe wurde durch längere Einwirkung von 12- bis 15 prozentiger Kalilauge bei Wasserbadtemperatur in Lösung gebracht, aus der filtrierten alkalischen Lösung durch verdünnte Schwefelsäure ausgefällt und schließlich durch Umkrystallisieren gereinigt.

\section{Methyläther des 1-Methyl-3-Phenyldioxindols.}

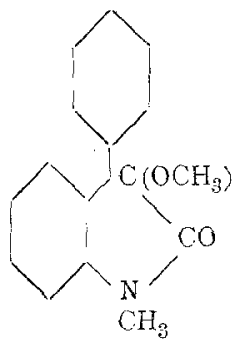

Das nach dem eben beschriebenen modifizierten Verfahre dargestellte Phenyldioxindol wird in zehnprozentiger Kalilauge gelöst und in einer Stöpselflasche mit Dimethylsulfat geschüttelt. Die Lösung erwärmt sich und bald fällt das Methylierungsprodukt ölig aus; das öl erstarrt beim weiteren Schütteln. Der so gewonnene Körper löst sich leicht in Methylalkohol. Durch zweimaliges Umkrystallisieren aus wässerigem Nethylalkohol resultieren blätterige Krystalle vom Schmelzpunkt $83^{\circ}$. 


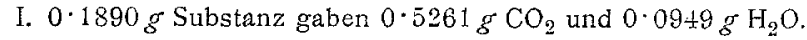

II. $0.1755 g$ Substanz gaben $0.4874 \mathrm{~g} \mathrm{CO}_{2}$ und $0.0932 \mathrm{~g} \mathrm{H}_{2} \mathrm{O}$.

III. $0.1804 \mathrm{~g}$ Substanz gaben $0.5023 \mathrm{~g} \mathrm{CO}_{2}$ und $0.0987 g \mathrm{H}_{2} \mathrm{O}$.

IV. $0.2021 \mathrm{~g}$ Substanz gaben in Ze is el's Apparat $0.1852 \mathrm{~g}$ Jodsilber.

$V .0 .2119 \mathrm{~g}$ Substanz gaben in $Z$ e is el's Apparat $0.1918 \mathrm{~g}$ Jodsilber.

In 100 Teilen:

\begin{tabular}{|c|c|c|c|c|c|}
\hline & & Gefunder & & & Berechnet für \\
\hline I. & II. & III. & IV. & V. & $\underbrace{\mathrm{C}_{16} \mathrm{H}_{15} \mathrm{NO}_{2}}$ \\
\hline C . . . . .75.92 & $75 \cdot 74$ & $75 \cdot 94$ & - & - & $75 \cdot 88$ \\
\hline$\ldots \ldots \ldots \quad 5.61$ & $5 \cdot 94$ & $6 \cdot 12$ & - & - & $5 \cdot 98$ \\
\hline $\mathrm{CH}_{3}$ an $\mathrm{O} \ldots-$ & - & - & $5 \cdot 84$ & $5 \cdot 77$ & $5 \cdot 93$ \\
\hline
\end{tabular}

\section{Acetylderivat des 3-Phenyldioxindols.}

In einem kleinen Kölbchen wurden $5 g$ Phenyldioxindo1 mit $20 \mathrm{~g}$ frisch destilliertem Essigsäureanhydrid 3 Stunden auf $145^{\circ} \mathrm{im}$ Ölbad erhitzt. Die klare rotbraune Lösung wurde in Wasser gegossen; es scheidet sich ein braunes Öl ab, das nach kurzer Zeit erstarrt. Der rotbraune Krystallbrei wurde trocken gesaugt und $z w e i m a l$ aus Benzol umkrystallisiert. Man erhält so die Substanz in kurzen säulenförmigen Kryställchen. Der Schmelzpunkt bleibt dann auch bei erneuertem Umkrystallisieren konstant bei $141^{\circ}$. Die roten Schmieren gehen schon beim ersten Umkrystallisieren vollkommen in die Mutterlauge. Die Elementaranalysen zeigen, daß der Körper ein Monoacetylderivat ist.

I. $0.1852 \mathrm{~g}$ Substanz gaben $0 \cdot 4880 \mathrm{~g} \mathrm{CO}_{2}$ und $0.0865 \mathrm{~g} \mathrm{H}_{2} \mathrm{O}$.

II. $0 \cdot 1618 \mathrm{~g}$ Substanz gaben $0.4271 \mathrm{~g} \mathrm{CO}_{2}$ und $0.0752 \mathrm{~g} \mathrm{H}_{2} \mathrm{O}$.

In 100 Teilen:

\begin{tabular}{|c|c|c|c|}
\hline & Gefu & den & Berechnet für \\
\hline & I & II & $\mathrm{C}_{16} \mathrm{H}_{13} \mathrm{NO}_{3}$ \\
\hline $\mathrm{C}$ & $71 \cdot 86$ & $71 \cdot 99$ & $71 \cdot 87$ \\
\hline$y$ & 5.22 & $5 \cdot 19$ & $4 \cdot 92$ \\
\hline
\end{tabular}


Methyläther des 1-Methyl-3-Benzyldioxindols.<smiles>CO[C@H]1C(=O)N(C)C2CC[IH]CC21CC1CCCCC1</smiles>

Die Gewinnung ist analog der des Phenylkörpers. Das Rohprodukt wird durch Umkrystallisieren aus wässerigem Methylalkohol gereinigt; es bildet bei $97^{\circ}$ schmelzende Nadeln.

I. $0 \cdot 1932 \mathrm{~g}$ Substanz gaben $0.5402 \mathrm{~g} \mathrm{CO}_{2}$ und $0.1099 \mathrm{~g} \mathrm{H}_{2} \mathrm{O}$.

II. $0.1827 \mathrm{~g}$ Substanz gaben $0.5094 \mathrm{~g} \mathrm{CO}_{2}$ und $0.1048 \mathrm{~g} \mathrm{H}_{2} \mathrm{O}$.

III. $0 \cdot 2586 \mathrm{~g}$ Substanz gaben in Z eise 1's Apparat $0 \cdot 2088 \mathrm{~g}$ Jodsilber.

IV. $0.2998 \mathrm{~g}$ Substanz gaben in Zeisel's Apparat $0.2495 \mathrm{~g}$ Jodsilber.

In 100 Teilen:

\begin{tabular}{|c|c|c|c|c|c|}
\hline & \multicolumn{4}{|c|}{ Gefunden } & \multirow{2}{*}{$\begin{array}{l}\text { Berechnet fir } \\
\mathrm{C}_{17} \mathrm{H}_{17} \mathrm{NO}_{2}\end{array}$} \\
\hline & 1. & II. & III. & IV. & \\
\hline$c \ldots \ldots$ & $.76 \cdot 25$ & $76 \cdot 02$ & - & - & $76 \cdot 35$ \\
\hline $\mathrm{H}$. & $.6 \cdot 36$ & $6 \cdot 41$ & - & - & $6 \cdot 43$ \\
\hline $\mathrm{CH}_{3}$ an $\mathrm{O}$. & - & - & $5 \cdot 15$ & $5 \cdot 31$ & $5 \cdot 63$ \\
\hline
\end{tabular}

Darstellung und Eigenschaften des 3-Methyldioxindols.

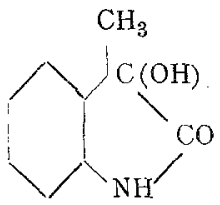

Die bei der Einwirkung von Methylmagnesiumjodid auf Isatin entstandene Doppelverbindung wird mit verdünnter Essigsäure zersetzt; das Methyldioxindol löst sich in der ätherischen Schicht und zum Teil in der wässerigen Lösung. Die ganze Flüssigkeit wird sofort von unveränderten Isatinteilchen durch Filtrieren befreit, sodann mehrmals ausgeäthert. 
Beim Einengen der vereinigten ätherischen Auszüge fällt ein Teil des Reaktionsproduktes gelb gefärbt krystallisiert aus; die ätherische Mutterlauge wird am Wasserbad eingedampft. Die beim Erkalten erstarrende dunkle Masse wird auf Tonteller gestrichen; das so erhaltene zimtbraune Pulver kann zur Darstellung des Methylierungsproduktes verwendet werden. Die gelben Krystalle werden aus Wasser unter Tierkohlezusatz umkrystallisiert und so rein weiß erhalten. Der Schmelzpunkt beträgt $160^{\circ}$. Die Substanz bildet glänzende körnige Kryställchen.

I. $0.1745 g$ Substanz gaben $0.4266 g \mathrm{CO}_{2}$ und $0.0874 g \mathrm{H}_{2} \mathrm{O}$.

II. $0.1775 \mathrm{~g}$ Substanz gaben $0.4305 \mathrm{~g} \mathrm{CO}_{2}$ und $0.0887 \mathrm{~g} \mathrm{H}_{2} \mathrm{O}$.

In 100 Teilen:

\begin{tabular}{|c|c|c|c|}
\hline & \multicolumn{2}{|c|}{ Gefunden } & \multirow{2}{*}{$\begin{array}{c}\text { Berechnet für } \\
\mathrm{C}_{9} \mathrm{H}_{9} \mathrm{NO}_{2}\end{array}$} \\
\hline & I & II & \\
\hline$c \ldots \ldots \ldots$ & $66 \cdot 67$ & $66 \cdot 15$ & $66 \cdot 21$ \\
\hline$H \ldots \ldots \ldots$ & $5 \cdot 60$ & $5 \cdot 59$ & $5 \cdot 57$ \\
\hline
\end{tabular}

\section{Methyläther des 1-Methyl-3-Methyldioxindols.}<smiles>CC(=O)N1C(C)C(C)C2CCCCC21</smiles>

Reines 3-Methyldioxindol wird in alkalischer Lösung mit Dimethylsulfat in der üblichen Weise behandelt. Das so erhaltene Produkt wird in wässerigem Methylalkohol gelöst und durch langsames Verdunsten in gut ausgebildeten würfelähnlichen Gebilden gewonnen, die bei $78.5^{\circ}$ schmelzen.

I. $0 \cdot 1860 \mathrm{~g}$ Substanz gaben $0 \cdot 4694 \mathrm{~g} \mathrm{CO}_{2}$ und $0.1128 \mathrm{~g} \mathrm{H}_{2} \mathrm{O}$.

I1. $0.1853 g$ Substanz gaben $0.4675 \mathrm{~g} \mathrm{CO}_{2}$ und $0.1141 \mathrm{~g} \mathrm{H}_{2} \mathrm{O}$.

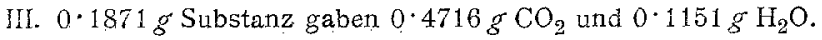

IV. $0.2162 \mathrm{~g}$ Substanz lieferten nach Dumas bei $20^{\circ} \mathrm{C}$. und $750 \mathrm{~mm}$ Barometerstand $14 \cdot 3 \mathrm{~cm}^{3}$ trockenen Stickstoff.

V. $0.2360 \mathrm{~g}$ Substanz gabèn nach Zeis e $10.2898 \mathrm{~g}$ Jodsilber.

VI. $0.2140 g$ Sutbstanz gaben nach $Z$ e ise $1.0 .2587 g$ Jodsilber. 
In 100 Teilen:

\begin{tabular}{|c|c|c|c|c|c|c|c|}
\hline \multirow{2}{*}{. } & \multicolumn{6}{|c|}{ Gefunden } & \multirow{2}{*}{$\begin{array}{r}\begin{array}{r}\text { Berechnet für } \\
\mathrm{C}_{11} \mathrm{H}_{19} \mathrm{NO}_{2}\end{array} \\
\end{array}$} \\
\hline & I. & II. & III. & IV. & V. & VI. & \\
\hline$\ldots \ldots$ & $.68 \cdot 83$ & $68 \cdot 81$ & $68 \cdot 74$ & - & - & - & $69 \cdot 06$ \\
\hline$H \ldots \ldots \ldots$ & $.6 \cdot 78$ & $6 \cdot 88$ & $6 \cdot 88$ & - & - & 一 & $6 \cdot 87$ \\
\hline$N \ldots \ldots$ & - & - & - & $7 \cdot 60$ & - & $\ldots$ & $7 \cdot 33$ \\
\hline $\mathrm{CH}_{3}$ an $\mathrm{O}$. & - & - & - & - & $7 \cdot 83$ & $7 \cdot 71$ & $7 \cdot 85$ \\
\hline
\end{tabular}

Bromprodukt des 3-Methyldioxindols.<smiles>CC1(O)C(=O)NC2CCC(Br)C1C2</smiles>

Reines 3-Methyldioxindol wird in wenig Wasser gelöst und mit der berechneten Menge gesättigten Bromwassers versetzt. Das Reaktionsprodukt fällt zuerst flockig aus und wandelt sich beim Stehen in eine fein krystallinische Modifikation um. Der Körper läßt sich aus Methylalkohol unter Zusatz von Tierkohle durch Verdunsten umkrystallisieren. Der in glitzernden, körnigen, rein weißen Kryställchen anschießende Körper bräunt sich beim Erhitzen bei $240^{\circ}$ und schmilzt zu einer rotbraunen Flüssigkeit bei 256 bis $257^{\circ}$.

I. $0.1857 \mathrm{~g}$ Substanz gaben $0 \cdot 3048 \mathrm{~g} \mathrm{CO}_{2}$ und $0 \cdot 0550 \mathrm{~g} \mathrm{H}_{2} \mathrm{O}$.

II. $0.1864 \mathrm{~g}$ Substanz gaben $0.3066 \mathrm{~g} \mathrm{CO}_{2}$ und $0.0536 \mathrm{~g} \mathrm{H}_{2} \mathrm{O}$.

In 100 Teilen:

\begin{tabular}{|c|c|c|}
\hline & Gefunden & \multirow{2}{*}{$\begin{array}{l}\text { Berechnet für } \\
\mathrm{C}_{9} \mathrm{H}_{8} \mathrm{O}_{2} \mathrm{NBr}\end{array}$} \\
\hline & II. & \\
\hline C $\ldots \ldots \ldots 44 \cdot 76$ & $44 \cdot 86$ & $44 \cdot 62$ \\
\hline $\mathrm{H} \ldots \ldots \ldots \ldots 3 \cdot 31$ & $3 \cdot 21$ & $3 \cdot 33$ \\
\hline
\end{tabular}

\section{Acetylierung des 3-Methyldioxindols.}

Diese Acetylierung wurde in derselben Weise vorgenommen wie beim Phenyldioxindol. Es wurde ebenfalls ein großer Überschuß an Essigsäureanhydrid angewendet. Beim Eingießen in Wasser scheidet sich sofort eine rötlich gefärbte Krystallmasse ab, die sich leicht aus wässerigem Alkohol um- 
krystallisieren läßt; nach zweimaligem Umkrystallisieren ist der Schmelzpunkt $125^{\circ}$; die Ausbeute ist nahezu theoretisch. Der Körper ist ein Diacetylderivat. Die Substanz bildet eine anscheinend blätterige Krystallmasse, unter dem Mikroskop sieht man jedoch ausgesprochen säulenförmige Krystalle.

I, $0 \cdot 1748 g$ Substanz gaben $0 \cdot 4039 g \mathrm{CO}_{2}$ und $0.0844 g \mathrm{H}_{2} \mathrm{O}$.

II. $0.1750 \mathrm{~g}$ Substan $z$ gaben $0.4031 \mathrm{~g} \mathrm{CO}_{2}$ und $0.0842 \mathrm{~g} \mathrm{H} \mathrm{H}_{2} \mathrm{O}$.

In 100 Teilen:

\begin{tabular}{|c|c|c|c|}
\hline & \multicolumn{2}{|c|}{ Gefunden } & \multirow{2}{*}{$\begin{array}{l}\text { Berechnet für } \\
\mathrm{C}_{13} \mathrm{H}_{13} \mathrm{NO}_{1}\end{array}$} \\
\hline & I. & 11. & \\
\hline$C \ldots$. & $63 \cdot 02$ & $62 \cdot 82$ & $63 \cdot 13$ \\
\hline$H \ldots \ldots$ & $5 \cdot 40$ & $5 \cdot 38$ & $5 \cdot 31$ \\
\hline
\end{tabular}

\section{Darstellung des 3-Phenyl-5-bromdioxindols aus 5-Bromisatin und Magnesiumphenylbromid.}<smiles>O=C(O)C1CCCC(NC2CCCC[IH]2)C1C(=O)O</smiles>

Die Darstellung erfolgt ganz analog der des Phenyldioxindols. Zur Trennung von unverändertem Bromisatin wurde das Präparat in Wasser vorsichtig aufgewirbelt. Das spezifisch schwerere Bromisatin scheidet sich am Boden ab, so daß man davon abgießen kann. Das Rohprodukt wird schließlich aus Alkohol umkrystallisiert. Man erhält so die Substanz in glänzenden, dünnen Stäbchen. Es schmilzt unter Zersetzung bei $243^{\circ}$ zu einer braunen Schmelze.

I. $0 \cdot 1874 g$ Substanz gaben $0.3793 g \mathrm{CO}_{2}$ und $0.0534 g \mathrm{H}_{2} \mathrm{O}$.

II. $0.1707 \mathrm{~g}$ Substanz gaben $0.3443 \mathrm{~g} \mathrm{CO}_{g}$ und $0.0498 \mathrm{~g} \mathrm{H}_{2} \mathrm{O}$.

In 100 Teilen:

\begin{tabular}{|c|c|c|c|}
\hline \multirow[t]{2}{*}{ texts } & \multicolumn{2}{|c|}{ Gefunden } & \multirow{2}{*}{$\begin{array}{l}\begin{array}{l}\text { Berechnet für } \\
\mathrm{C}_{14} \mathrm{H}_{10} \mathrm{O}_{2} \mathrm{NBr}\end{array} \\
\end{array}$} \\
\hline & I. & II. & \\
\hline$c \ldots .$. & $55 \cdot 20$ & $55 \cdot 01$ & $55 \cdot 26$ \\
\hline$H \ldots \ldots$ & $3 \cdot 18$ & $3 \cdot 26$ & $3 \cdot 32$ \\
\hline
\end{tabular}




\section{Darstellung des 3-Methyl-5-bromdioxindols aus Bromisatin und Magnesiummethyljodid.}<smiles>CC1CC(Br)CCC1NC(=O)O</smiles>

Die Reaktion verlauft ganz analog wie sonst. Das Reaktionsprodukt ist im Gegensatz zum Methyldioxindol in Wasser unlöslich. Es wird zur Reinigung aus Alkohol umkrystallisiert.

I. $0.1883 \mathrm{~g}$ Substanz gaben $0.3058 \mathrm{~g} \mathrm{CO}_{2}$ und $0.0556 \mathrm{~g} \mathrm{H}_{2} \mathrm{O}$.

Il. $0.2189 \mathrm{~g}$ Substanz gaben $0.3589 \mathrm{~g} \mathrm{CO}$, und $0.0673 g \mathrm{H}_{2} \mathrm{O}$.

In 100 Teilen:

\begin{tabular}{|c|c|c|c|}
\hline & \multicolumn{2}{|c|}{ Gefunden } & \multirow{2}{*}{$\begin{array}{l}\begin{array}{c}\text { Berechnet für } \\
\mathrm{C}_{9} \mathrm{H}_{8} \mathrm{NO}_{2} \mathrm{Br}\end{array} \\
\end{array}$} \\
\hline & I. & II. & \\
\hline$C \ldots \ldots$ & $\pm 4 \cdot 29$ & $44 \cdot 71$ & $44 \cdot 62$ \\
\hline $\mathrm{H} \ldots \ldots$ & $3 \cdot 30$ & $3 \cdot 44$ & $3 \cdot 33$ \\
\hline
\end{tabular}

Der Körper bräunt sich beim Erhitzen bei $240^{\circ}$ und schmilzt zu einer rotbraunen Flüssigkeit bei $258^{\circ}$. Die Substanz verhält sich mithin beim Erhitzen in der Kapillare genau so wie das Bromprodukt des 3-Methyldioxindols. Auch ein fein verriebenes Gemenge beider Substanzen bräunt sich bei ungefähr $240^{\circ}$ und schmilzt bei $258^{\circ} \mathrm{zu}$ einer klaren rotbraunen Flüssigkeit.

\section{Methyläther des 1-Methyl-3-Methyl-5-Bromdioxindols.}

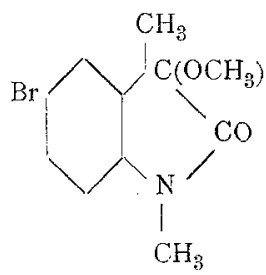

Die aus wässerigem Methylalkohol umkrystallisierte Substanz schmilzt bei $142^{\circ}$. Sie bildet nadelige Krystalle. 
916 M. Kohn und A. Ostersetzer, Abkömmlinge des Dioxindols.

I. $0.1700 \mathrm{~g}$ Substanz gaben $0.3034 \mathrm{~g} \mathrm{CO}_{2}$ und $0.0713 \mathrm{~g} \mathrm{H} \mathrm{H}_{2} \mathrm{O}$.

Il. $0.1790 \mathrm{~g}$ Substanz gaben $0.3187 \mathrm{~g} \mathrm{CO}_{2}$ und $0.0726 \mathrm{~g} \mathrm{H}_{2} \mathrm{O}$.

III. $0 \cdot 2554 \mathrm{~g}$ Substanz gaben nach $Z$ eis e $10 \cdot 2208 \mathrm{~g}$ Jodsilber.

In 100 Teilen:

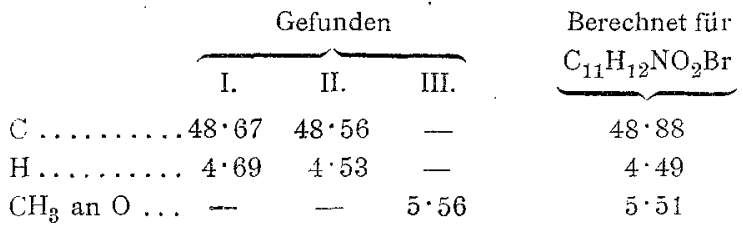

Um die Identität des Reaktionsproduktes aus Bromisatin und Methylmagnesiumjodid mit dem Bromierungsprodukt des 3-Methyldioxindols zu erweisen, wurde auch letzteres mitteis Kali- und Dimethylsulfat methyliert. Es resultierte ein rein weißes Reaktionsprodukt, welches durch Umkrystallisieren aus wässerigem Methylalkohol in glänzenden nadeligen Krystallen erhalten wurde und den Schmelzpunkt $142^{\circ}$ zeigte.

Die Analyse ergab:

$0.1763 g$ Substanz lieferten $0.3133 g \mathrm{CO}_{2}$ und $0.0726 \mathrm{~g} \mathrm{H}_{2} \mathrm{O}$.

In 100 Teilen:

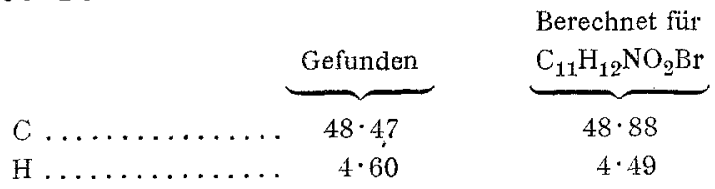

Der Mischschmelzpunkt dieser Substanz mit dem Dimethylderivat des Methybromdioxindols zeigte keine Spur einer Depression. Das Gemenge war bei $144^{\circ}$ völlig verflüssigt. 